\title{
O USO DE BENZODIAZEPÍNICOS EM MULHERES IDOSAS E O PAPEL DO MEDICO DA ATENÇÃO PRIMÁRIA
}

\author{
Alexandre Carvalho ${ }^{1}$, Luciana Farias dos Santos ${ }^{1}$, Simone Shirasaki Orosco ${ }^{1}$ \\ Universidade do Oeste Paulista - UNOESTE, Faculdade de Medicina, Presidente Prudente, SP. e-mail: \\ alee.carvalho@live.com
}

\section{RESUMO}

O uso de benzodiazepínicos é frequente em idosos do gênero feminino. Essa situação é preocupante, na medida em que esse segmento populacional apresenta maior predisposição aos efeitos adversos e à toxicidade dessas medicações. Assim, este estudo teve como objetivo identificar o papel do médico da atenção primária diante do uso inadequado de benzodiazepínico em idosas. Esta pesquisa utilizou como metodologia a revisão integrativa. Os artigos foram comparados e agrupados por similaridade de conteúdo, sendo construídas duas categorias para análise: uso inadequado de benzodiazepínicos em idosas e papel do médico da atenção primária. Conclui-se que se deve instituir o uso racional de benzodiazepínicos na população idosa, além disso, o médico precisa saber identificar tolerância e dependência e como manejar esses pacientes.

Palavras-chave: ansiolíticos, idoso, mulheres, atenção primária à saúde, medicina comunitária.

\section{THE USE OF BENZODIAZEPINES IN ELDERLY WOMEN AND THE ROLE OF THE DOCTOR OF PRIMARY CARE.}

\begin{abstract}
The use of benzodiazepines is common in female elderly. This situation is disturbing to the extent that this population segment has a greater predisposition to adverse effects and toxicity of those drugs. This study aims to identify the role of the primary care physician on the inappropriate use of benzodiazepines in elderly. This study used as methodology the integrative review. The articles were compared and grouped by similarity of content, being built two categories for analysis: inappropriate use of benzodiazepines in elderly and role of the primary care physician. The conclusion that must provide the rational use of benzodiazepines in the elderly, in addition, the doctor needs to know how to identify tolerance and dependence and how to manage these patients.
\end{abstract}

Keywords: anti-anxiety agents, aged, women, primary health care, community medicine.

\section{INTRODUÇÃO}

Os benzodiazepínicos (BZD) são fármacos que apareceram no mercado na década de 60 e são caracterizados por seus efeitos ansiolíticos, quando em doses baixas, sedativos e hipnóticos em doses altas, além de possuir propriedades miorrelaxantes e anticonvulsivantes ${ }^{2,9}$. A partir de seu surgimento, a prescrição e consumo desse grupo de psicofármaco alcançaram níveis impressionantes estando hoje entre as drogas mais vendidas no mundo'. Estima-se que o consumo dessas medicações dobre a cada cinco anos ${ }^{2}$.

O consumo crescente dos benzodiazepínicos pode ser resultado de um período turbulento que tem caracterizado as últimas décadas, associado à diminuição da resistência da comunidade ao estresse, à introdução de novas drogas no mercado, pressão propagandística das indústrias farmacêuticas e a prescrição médica inadequada ${ }^{2}$.

No Brasil, a taxa semanal de consumo de tranquilizantes na população em geral é de $8 \%$, a maioria por prescrição de médicos generalistas enquanto no Chile, um estudo em Santiago revelou uma prevalência anual no uso de psicofármacos de $31,4 \%$, sendo a maioria mulheres $(38,9 \%)^{3}$.

Existe o risco de que com uma indicação inadequada, os pacientes usuários destas substâncias aumentem as doses sem comunicar ao médico, entre outras razões pela tolerância que estes geram ${ }^{4}$. A eficácia dos benzodiazepínicos é bem descrita nos 
tratamentos de curta duração, porém, o uso prolongado é contraindicado devido aos riscos de efeitos adversos, incluindo a dependência ${ }^{3}$. Se usado por até três meses, o risco é praticamente nulo, enquanto que de três a 12 meses é de $10 \%$ a $15 \%$ e por mais de 12 meses é de $25 \%$ a $40 \%{ }^{2}$.

No Brasil, mesmo com a exigência de retenção de receita médica pela dispensação, o controle do uso desse grupo de medicamento ainda é falho resultando em automedicação, uso sem supervisão e prejuízo para o próprio paciente ${ }^{5}$. Reflexo disso, é o fato de o país ser o quinto consumidor de diazepam no mundo e o segundo de clonazepam ${ }^{12}$.

O uso de benzodiazepínicos é frequente em idosos, o número de usuários aumenta conforme a idade e as mulheres integram o grupo mais associado ao uso dessas drogas.

Essa situação é preocupante, na medida em que os idosos apresentam maior predisposição aos efeitos adversos e à toxicidade dessas medicações, tais como risco de quedas, déficit cognitivo, dependência, sonolência diurna e perda da memória ${ }^{1,5,6}$. Uma revisão de ensaios clínicos demonstrou que a eficácia dos benzodiazepínicos no tratamento da insônia em idosos não institucionalizados não é isenta de riscos mesmo em curto prazo, e não houve constatação da efetividade do tratamento se em longo prazo ${ }^{1,2}$.

Poucos estudos mostram que o uso de BZD por mulheres idosas é um problema de saúde pública relevante, o que demonstra a necessidade de mais estudos que estimem o risco de eventos adversos associado ao uso concomitante de benzodiazepínicos e de polifarmácia em idosos ${ }^{5}$.

Nesse contexto, os médicos da família, pela sua posição na rede de cuidados em saúde, podem detectar e lidar com os sintomas psiquiátricos precocemente em pacientes que vêm a sua consulta com transtorno de ansiedade, assim como identificar e manejar pacientes usuários crônicos de BZDs ${ }^{7}$.

A fim de contribuir com a investigação sobre a temática, o presente estudo teve como objetivo conhecer as produções científicas que abordaram o papel do médico da atenção primária diante do uso inadequado de benzodiazepínico em idosas.

\section{METODOLOGIA}

A metodologia de análise consistiu de uma revisão integrativa ${ }^{8}$, cuja finalidade foi reunir e sintetizar resultados de pesquisas, de maneira sistemática e ordenada, para o aprofundamento do conhecimento a respeito do papel do médico da Atenção Primária em Saúde diante do uso inadequado de benzodiazepínicos em idosas.

Para a coleta de dados foram selecionados artigos publicados no período de abril de 2000 a junho de 2015, indexados na base de dados Scientific Eletronic Library Online (SCiELO) e Literatura Latino-Americana e do Caribe em Ciências da Saúde (LILACS). Os descritores empregados para a busca foram "idoso", "dependência", "benzodiazepina" e "atenção primária à saúde".

Foram considerados para a análise dos estudos encontrados nesta revisão: o tipo de estudo e método, os objetivos, os resultados e as conclusões. Para a seleção dos artigos ocorreu primeiramente a análise dos títulos e resumos, em uma segunda etapa houve uma avaliação do texto completo. Foram incluídos os artigos em língua portuguesa e espanhola, na íntegra e que ressaltaram a temática referente ao uso de benzodiazepínico na população idosa e o papel do médico da atenção primária. Foram excluídos os artigos que não relatavam nenhum envolvimento com o tema e os artigos que não disponibilizavam o resumo para verificação. Os artigos foram comparados e agrupados por similaridade de conteúdo, sob a forma de categorias empíricas, sendo construídas duas categorias para análise: uso inadequado de benzodiazepínicos em idosas e papel do médico da atenção primária.

\section{RESULTADOS}

Foram identificados 148 artigos para a revisão Integrativa, 34 na SciELO e 104 no LILACS. Com os descritores "benzodiazepina" + "dependência" encontraram-se 21 resultados selecionando-se 4 artigos, "benzodiazepina" + "idoso", encontraram-se 13 e selecionaram-se 3, "benzodiazepina" + "anciano" encontraram-se 2 e selecionaram-se 1, e "benzodiazepine" + "aged" encontraram-se 102 e selecionaram-se 5, com "atenção primária à saúde" + "benzodiazepina" encontraram-se 5 e selecionaram-se 2. Após a seleção por títulos, resumos disponíveis e ano de publicação (período de 2000 a 2015), permaneceram 13 artigos. Deste grupo foram excluídos 4 por não possuírem relação com o tema. Foram para leitura integral 9 artigos.

Os resultados dos principais artigos selecionados estão apresentados no Quadro 1. 
Quadro 1. Artigos selecionados.

\begin{tabular}{|c|c|c|c|}
\hline $\begin{array}{c}\text { Autor, } \\
\text { ano }\end{array}$ & $\begin{array}{c}\text { Tipo de } \\
\text { Estudo/Método }\end{array}$ & Objetivos & Resultados e Conclusões \\
\hline $\begin{array}{l}\text { Huf et } \\
\text { al., } 2000\end{array}$ & $\begin{array}{l}\text { O padrão de uso de } \\
\text { benzodiazepínicos e } \\
\text { os fatores associados } \\
\text { ao uso prolongado } \\
\text { destes foram } \\
\text { examinados com } \\
\text { base em um } \\
\text { questionário } \\
\text { padronizado, } \\
\text { aplicado a } 634 \\
\text { mulheres com mais } \\
\text { de sessenta anos, } \\
\text { participantes de um } \\
\text { centro de } \\
\text { convivência para } \\
\text { idosos no Rio de } \\
\text { Janeiro, entre maio } \\
\text { de } 1992 \text { e dezembro } \\
\text { de } 1995 \text {. }\end{array}$ & $\begin{array}{l}\text { Avaliar o } \\
\text { padrão de } \\
\text { utilização de } \\
\text { benzodiazepínic } \\
\text { os numa } \\
\text { população que } \\
\text { apresenta } \\
\text { riscos especiais: } \\
\text { as mulheres } \\
\text { com mais de } \\
\text { sessenta anos. }\end{array}$ & $\begin{array}{l}\text { A prevalência de uso de benzodiazepínicos na última } \\
\text { quinzena foi estimada em } 21,3 \% \text { (IC } 95 \% 18,1-24,5) \text {, e } \\
\text { a prevalência de uso diário por } 12 \text { meses ou mais em } \\
7,4 \% \text { (IC } 95 \% 5,4-9,4 \text { ). Em uma análise multivariada, o } \\
\text { número de medicamentos consumidos mostrou } \\
\text { associação importante e progressiva com o uso } \\
\text { prolongado de benzodiazepínicos, com OR }=2,77 \text { (IC } \\
95 \% 1,17-6,57 \text { ) para aquelas que consomem entre } \\
\text { quatro a seis medicamentos e OR = 7,62 (IC } 95 \% 3,18- \\
18,26 \text { ) para aquelas que consomem mais de sete } \\
\text { medicamentos. Queixas de insônia (OR = 8,87 IC } 95 \% \\
2,53-31,06 \text { ) e cefaleia (OR }=3,53 \text { IC } 95 \% 1,82-6,89 \text { ) } \\
\text { também estiveram fortemente associadas a este } \\
\text { padrão de uso. Os achados apontam para a } \\
\text { necessidade de estudos em que os pacientes que } \\
\text { iniciam o uso de benzodiazepínicos possam ser } \\
\text { acompanhados ao longo do tempo. Estudos } \\
\text { longitudinais permitirão aferir com maior acurácia o } \\
\text { tempo de uso e explorar os fatores envolvidos. }\end{array}$ \\
\hline $\begin{array}{l}\text { Silva } \\
\text { Herrera } \\
\text { et al. } \\
2002\end{array}$ & $\begin{array}{l}\text { Realizou-se um } \\
\text { estudo descritivo, } \\
\text { observacional, de } \\
\text { prescrição/indicação } \\
\text { de medicamentos } \\
\text { durante os meses de } \\
\text { novembro de } 1999 \text { a } \\
\text { janeiro de } 2000 \text { na } \\
\text { Cidade de Havana, } \\
\text { utilizando-se } \\
\text { questionário } \\
\text { padronizado aplicado } \\
\text { a } 804 \text { consumidores } \\
\text { de } \\
\text { benzodiazepínicos. }\end{array}$ & $\begin{array}{l}\text { Estimar a } \\
\text { prevalência do } \\
\text { uso crônico de } \\
\text { benzodiazepina } \\
\text { s entre os } \\
\text { pacientes } \\
\text { adultos, } \\
\text { determinar as } \\
\text { características } \\
\text { socioculturais, } \\
\text { os motivos da } \\
\text { prescrição, as } \\
\text { drogas mais } \\
\text { utilizadas o } \\
\text { nível de }\end{array}$ & $\begin{array}{l}\text { Do total de } 804 \text { pessoas entrevistadas, consumidoras } \\
\text { de benzodiazepinas, maiores de } 15 \text { anos de idade } 57 \% \\
\text { o faziam de forma crônica. Desta população } \\
\text { consumidora crônica } 73,6 \% \text { são do sexo feminino, com } \\
\text { predomínio entre os maiores de } 60 \text { anos de idade, } \\
\text { donas de casa, de menores níveis educacionais e de } \\
\text { estado civil casado. Comprovou-se que a } \\
\text { benzodiazepina mais utilizada foi o diazepam, } \\
\text { observado em } 65,9 \% \text { dos casos. A maioria das } \\
\text { prescrições foi realizada para a ansiedade }(57,7 \%) \text { e } \\
\text { insônia ( } 31,4 \%) \text {. A automedicação ocorreu em } 25,1 \% \\
\text { dos consumidores crônicos. }\end{array}$ \\
\hline $\begin{array}{l}\text { Galleguill } \\
\text { os et al. } \\
2003\end{array}$ & $\begin{array}{l}\text { Realizou-se um } \\
\text { estudo transversal } \\
\text { durante os dias } 6 \text { e } 7 \\
\text { de setembro do ano } \\
\text { de } 2000 \text {, em } \\
\text { consultórios de } \\
\text { atenção primária em } \\
\text { zonas urbanas e } \\
\text { rurais de Santiago, } \\
\text { aplicando-se } \\
\text { questionário a } 188 \\
\text { pacientes com mais } \\
\text { de } 15 \text { anos. }\end{array}$ & $\begin{array}{l}\text { Verificar a } \\
\text { frequência de } \\
\text { uso de } \\
\text { benzodiazepínic } \\
\text { os entre } \\
\text { pacientes } \\
\text { (idade média de } \\
46,8 \text { ) de } \\
\text { cuidados } \\
\text { primários. }\end{array}$ & $\begin{array}{l}\text { A amostra foi constituída por } 188 \text { pessoas, } 65,7 \% \\
\text { mulheres e } 34,3 \% \text { homens. A idade média foi de } \\
46,8 \pm 17,5 \text { anos. Em } 31,5 \% \text { dos entrevistados utilizava } \\
\text { algum tipo de psicofármaco em algum momento do } \\
\text { estudo. Os benzodiazepínicos corresponderam a } 82,1 \% \\
\text { do total de psicofármacos. A prevalência anual de } \\
\text { consumo de benzodiazepínicos foi de } 26 \% \text { ( } 46 \\
\text { sujeitos). A média de tempo de consumo entre os } \\
\text { usuários atuais desta amostra foi de } 3,2 \text { anos ( } 38 \\
\text { meses). A prescrição do fármaco foi feita em } 74,5 \% \\
\text { por um médico generalista, em } 12,8 \% \text { por um } \\
\text { psiquiatra, e em } 6,4 \% \text { por outro especialista. Em } 6,4 \% \\
\text { da amostra consumiam benzodiazepínicos sem } \\
\text { prescrição médica. }\end{array}$ \\
\hline
\end{tabular}




\begin{tabular}{|c|c|c|c|}
\hline $\begin{array}{l}\text { García et } \\
\text { al. } 2003\end{array}$ & $\begin{array}{l}\text { Realizou-se uma } \\
\text { revisão bibliográfica }\end{array}$ & $\begin{array}{l}\text { O objetivo foi } \\
\text { fazer referência } \\
\text { a alguns } \\
\text { benzodiazepínic } \\
\text { os disponíveis } \\
\text { na atualidade } \\
\text { assim como } \\
\text { suas } \\
\text { características } \\
\text { farmacológicas } \\
\text { e uso. }\end{array}$ & $\begin{array}{l}\text { Quase todos os efeitos dos BZD se devem à inibição do } \\
\text { SNC, através de potencialização do GABA. Devido a } \\
\text { maior margem de segurança em relação aos } \\
\text { medicamentos antigos, substituição amplamente às } \\
\text { outras classes para o tratamento da ansiedade e } \\
\text { insônia. Os fármacos de eleição para a insônia são } \\
\text { aqueles de ação curta. Para evitar dependência física o } \\
\text { médico deve usar dose mínima e eficaz, fazer } \\
\text { tratamentos curtos e intermitentes, suspender a } \\
\text { medicação assim que se controlem os sintomas, o } \\
\text { tratamento não deve ser maior que } 2 \text { meses. Quando } \\
\text { o objetivo é ansiolítico recomendam-se os BZD de } \\
\text { meia vida prolongada. Esses fármacos são uma } \\
\text { ferramenta valiosa, mas devem-se levar em } \\
\text { consideração os efeitos colaterais e a dependência } \\
\text { para seu uso. }\end{array}$ \\
\hline $\begin{array}{l}\text { Auchews } \\
\text { ki et al. } \\
2004\end{array}$ & $\begin{array}{l}\text { Entrevistaram-se } 120 \\
\text { pacientes, sendo } 39 \\
\text { homens e } 81 \\
\text { mulheres os quais } \\
\text { procuraram } \\
\text { farmácias em } \\
\text { Curitiba para } \\
\text { comprar } \\
\text { benzodiazepínicos, } \\
\text { aplicando-se } \\
\text { questionário pré- } \\
\text { estabelecido. }\end{array}$ & $\begin{array}{l}\text { Avaliar as } \\
\text { orientações } \\
\text { médicas } \\
\text { recebidas com } \\
\text { relação aos } \\
\text { principais } \\
\text { efeitos } \\
\text { colaterais dos } \\
\text { medicamentos. }\end{array}$ & $\begin{array}{l}\text { Treze por cento relataram terem sido orientados sobre } \\
\text { os } 3 \text { principais efeitos colaterais do medicamento, } 27 \% \\
\text { a respeito de pelo menos dois e } 40 \% \text { apenas de um, } \\
\text { enquanto que } 19 \% \text { não recebeu nenhuma orientação. } \\
\text { Houve predomínio da orientação não beber ( } 85 \%) \text {, } \\
\text { seguida do cuidado para operar maquinas e dirigir } \\
\text { veículos ( } 46 \% \text { e por ultimo o risco de } \\
\text { desenvolvimento da dependência ( } 31 \%) \text {. Sessenta e } \\
\text { um por cento utilizavam por mais de um ano, } 94 \% \\
\text { tiveram insucesso na interrupção do medicamento, e } \\
22 \% \text { tiveram pouca orientação sobre o uso do } \\
\text { medicamento. }\end{array}$ \\
\hline $\begin{array}{l}\text { Minaya } \\
\text { et al. } \\
2009\end{array}$ & $\begin{array}{l}\text { Em uma amostra de } \\
39 \text { pacientes do } \\
\text { instituto Nacional de } \\
\text { Psiquiatria Ramon de } \\
\text { La Fuente na cidade } \\
\text { do México. O } \\
\text { diagnóstico de } \\
\text { dependência a } \\
\text { benzodiazepínicos foi } \\
\text { realizado a partir do } \\
\text { SCID-I, a gravidade } \\
\text { da depressão se } \\
\text { avaliou mediante a } \\
\text { escala de } \\
\text { Montgomery e } \\
\text { Asberg, os sintomas } \\
\text { da ansiedade com a } \\
\text { escala de Hamilton. }\end{array}$ & $\begin{array}{l}\text { Descrever e } \\
\text { comparar as } \\
\text { características } \\
\text { sociodemografi } \\
\text { cas e clinicas } \\
\text { relacionadas } \\
\text { com a } \\
\text { prescrição e } \\
\text { com o uso de } \\
\text { benzodiazepínic } \\
\text { os em adultos } \\
\text { idosos com e } \\
\text { sem } \\
\text { dependência } \\
\text { aos mesmos em } \\
\text { um serviço } \\
\text { especializado } \\
\text { de } \\
\text { psicogeriatria. }\end{array}$ & $\begin{array}{l}\text { Do total da amostra, } 92,3 \% \text { eram mulheres, com idade } \\
\text { média de } 70,8 \text { e desvio padrão de } 5,8 \text {. O nível de } \\
\text { escolaridade média foi de } 7 \text { anos e a principal } \\
\text { ocupação foi atividade do lar ( } 82 \% \text { ) seguida de } \\
\text { desemprego (10\%) e emprego remunerado ( } 7 \%) \text {. Dos } \\
\text { pacientes, } 38 \text { tinha como diagnostico principal o } \\
\text { transtorno depressivo maior. Cinquenta e três por } \\
\text { cento completaram critérios para diagnostico de } \\
\text { transtorno de ansiedade. A indicação médica principal } \\
\text { (59\%) foi a presença de transtorno de ansiedade, } \\
\text { seguida de insônia (41\%). A idade de inicio foi de } 57,5 \\
\text { anos, em média, com tempo médio de } 625 \text { semanas. } \\
\text { Sessenta e nove por cento dos entrevistados } \\
\text { apresentavam dependência aos benzodiazepínicos. A } \\
\text { depressão em idosos relacionada com a ansiedade não } \\
\text { deveria ser tratada com benzodiazepínicos devido ao } \\
\text { risco de dependência e a relação com a intensidade } \\
\text { dos sintomas de depressão. }\end{array}$ \\
\hline
\end{tabular}




\begin{tabular}{|c|c|c|c|}
\hline $\begin{array}{l}\text { Oliveira, } \\
2009\end{array}$ & $\begin{array}{l}\text { Estudo descritivo, } \\
\text { com uma amostra } \\
\text { populacional de } 1081 \\
\text { pacientes de um } \\
\text { consultório no } \\
\text { município de } \\
\text { Santiago, cujos dados } \\
\text { foram extraídos do } \\
\text { censo semestral do } \\
\text { Programa de Saúde } \\
\text { Mental entre } \\
\text { primeiro de janeiro a } \\
30 \text { de junho de } 2005 .\end{array}$ & $\begin{array}{l}\text { Determinar a } \\
\text { prevalência de } \\
\text { dependência e } \\
\text { benzodiazepínic } \\
\text { os e descrever } \\
\text { características } \\
\text { sociodemografi } \\
\text { cas da amostra. }\end{array}$ & $\begin{array}{l}\text { Do total de } 1081 \text { pacientes, } 111 \text { foram diagnosticados } \\
\text { como dependentes de benzodiazepínicos pelos } \\
\text { critérios do DSM-IV. A idade média foi de } 60 \text { anos, com } \\
\text { uma proporção de sexos de } 3 \text { mulheres para cada } \\
\text { homem. Em } 66 \% \text { deste grupo, havia pelo menos uma } \\
\text { patologia mental associada ( } 20 \% \text { transtorno do animo, } \\
12 \% \text { transtorno de ansiedade generalizada, } 7 \% \\
\text { transtorno do pânico, } 15 \% \text { transtorno misto } \\
\text { ansiedade-depressão, } 11 \% \text { transtorno de } \\
\text { personalidade). Deve-se enfrentar o problema com o } \\
\text { diagnostico da severidade do problema, realizar } \\
\text { desintoxicação gradual nos casos de melhor } \\
\text { prognostico, indicar BZD com precaução, desenvolver } \\
\text { e manter vínculo terapêutico sólido. }\end{array}$ \\
\hline $\begin{array}{l}\text { Ricardo e } \\
\text { Gárciga, } \\
2010\end{array}$ & $\begin{array}{l}\text { Estudo descritivo de } \\
\text { corte transversal, } \\
\text { realizado no segundo } \\
\text { semestre de 2007, } \\
\text { em consultório } \\
\text { pertencente a área } \\
\text { de saúde da } \\
\text { Policlínica de Campo } \\
\text { Florido no Município } \\
\text { de Havana, sobre o } \\
\text { comportamento do } \\
\text { consumo dos } \\
\text { benzodiazepínicos, } \\
\text { em uma amostra ao } \\
\text { acaso de } 79 \\
\text { pacientes, todos } \\
\text { maiores de } 60 \text { anos. }\end{array}$ & $\begin{array}{l}\text { Examinar o } \\
\text { consumo de } \\
\text { benzodiazepínic } \\
\text { os em pacientes } \\
\text { da terceira } \\
\text { idade, } \\
\text { analisando } \\
\text { variáveis de } \\
\text { idade, sexo, } \\
\text { prescrição } \\
\text { facultativa, } \\
\text { motivo de } \\
\text { prescrição. }\end{array}$ & $\begin{array}{l}\text { Da amostra, } 74,6 \% \text { eram mulheres, o grupo de idade } \\
\text { que predominou foi de } 60-64 \text { anos com } 50,6 \% \text { do total. } \\
\text { O motivo principal para o consumo do fármaco foi } \\
\text { ansiedade }(54,5 \%) \text {, em segundo lugar a insônia (29\%). } \\
\text { Conclui-se que a prescrição de psicofármacos na } \\
\text { população de terceira idade é uma pratica clinica } \\
\text { habitual que merece atenção. }\end{array}$ \\
\hline $\begin{array}{l}\text { Souza et } \\
\text { al., } 2013\end{array}$ & $\begin{array}{l}\text { Foi realizado um } \\
\text { estudo qualitativo } \\
\text { com amostra de } 33 \\
\text { mulheres entre } 18 \text { a } \\
60 \text { anos que } \\
\text { utilizaram uso } \\
\text { indevido de } \\
\text { benzodiazepínicos no } \\
\text { último ano, para } \\
\text { insônia e ansiedade } \\
\text { por mais de } 4 \\
\text { semanas. A coleta de } \\
\text { dados foi através de } \\
\text { entrevista } \\
\text { semiestruturada. }\end{array}$ & $\begin{array}{l}\text { Compreender } \\
\text { qualitativament } \\
\text { e crenças e } \\
\text { valores } \\
\text { associados ao } \\
\text { consumo } \\
\text { indevido de } \\
\text { benzodiazepínic } \\
\text { os por } \\
\text { mulheres. }\end{array}$ & $\begin{array}{l}\text { A maioria das entrevistadas referiu tempo de uso bem } \\
\text { superior ao recomendado (mediana: } 7 \text { anos) e compra } \\
\text { com receita médica. Os motivos de uso mais citados } \\
\text { foram diminuição da ansiedade, problemas de insônia } \\
\text { e fuga dos problemas. Apesar de reconhecerem a } \\
\text { possibilidade de dependência, esta não motivou a } \\
\text { interrupção do uso. O acompanhamento médico não } \\
\text { pareceu, necessariamente, estimular a percepção de } \\
\text { risco dos benzodiazepínicos, sendo um fator que } \\
\text { favoreceu a manutenção do uso prolongado. }\end{array}$ \\
\hline
\end{tabular}

\section{DISCUSSÃO}

Uso inadequado de benzodiazepínicos em idosas

$O$ uso inadequado de BZD, definido como

o uso sem supervisão médica ou em quantidades/prazos superiores ao preconizado para tratamento, tem sido crescente nos últimos anos, tornando-se objeto de preocupação na área de saúde pública junto com outros medicamentos psicotrópicos 5 . Segundo Minaya et al. $^{6}$, o uso de benzodiazepínicos para o tratamento de ansiedade e insônia não deve ultrapassar período maior que quatro semanas, 
enquanto Fernández García et al. ${ }^{7}$, recomendam no máximo 2 meses.

É de extrema relevância elucidar o paciente sobre o problema que o mesmo possui, a medicação que esta sendo usada e seus efeitos colaterais, assim como estabelecer um plano terapêutico com prazo adequado e manter vinculo estável com 0 cliente ${ }^{5}$ Os benzodiazepínicos trazem como efeitos colaterais principais diminuição da atenção, interação com o álcool e risco potencial de dependência e tolerância ${ }^{2}$. Em especial os idosos, os quais já possuem maior risco de queda, tem risco adicional quando fazem uso dessa classe de droga'.

A grande maioria dos artigos traz como característica sociodemográfica principal, em relação ao uso do medicamento, o predomínio do sexo feminino, o que se considera como fator de risco para dependência e uso prolongado, junto ao aumento da idade ${ }^{1,3,4,9,12}$. Pode-se atribuir isso ao maior espaço que a mulher passou a ocupar na sociedade moderna, agregando tarefas àquelas que já possuíam no cuidado de casa e da família, tornando-a susceptível ao estresse e ansiedade ${ }^{5}$.

Nota-se, também, que há maior uso na terceira idade, uma faixa etária em que devido ao processo natural de desenvolvimento e diminuição do metabolismo de drogas, pode haver superdosagem com toxicidade e maior vulnerabilidade a efeitos colaterais ${ }^{3,6,7,10}$.

A qualidade de vida de um usuário de benzodiazepínico pode ser prejudicada pelo uso indevido, repercutindo nas atividades diárias com diminuição da cognição, da independência e da autonomia. Floriano e Dalgalarrondo ${ }^{11}$ descrevem um risco 12 vezes maior de haver pior qualidade de vida física quando há uso dessa classe, com queixas como diminuição da energia física, sono insatisfatório, dificuldade na locomoção e maior busca do atendimento médico.

Evidencia-se a falta de orientação dos pacientes, pois a maioria não tem a percepção do risco a que é submetido quando usando a medicação, não são informados sobre a possibilidade de dependência e o reconhecimento da mesma, e também não são orientados sobre a interrupção quando controlados os sintomas os quais motivaram a prescrição ${ }^{1,5}$. Estudo realizado em Santiago demonstrou que o tempo médio de consumo foi de 3,2 anos $^{4}$.

\section{Papel do médico da atenção primária}

O médico da atenção primária deve ficar atento à idade avançada do paciente e o uso prolongado, as queixas de sedação diurna e à meia vida da droga utilizada, pois são fatores de risco para o aumento da toxicidade e desenvolvimento de dependência ${ }^{6}$. O retorno do paciente ao médico periodicamente é um fator de importância para o monitoramento da dose, avaliação dos efeitos colaterais e da resposta terapêutica $^{1,2}$.

A literatura tem apontado que nos indivíduos cuja queixa é a insônia crônica, recomenda-se orientar o cliente a definir um horário fixo constante e dormir mais tarde, desestimular o consumo de substâncias estimulantes próximo ao horário de dormir, assim como ingerir carnes vermelhas e comidas pesadas durante a noite, evitar ruídos incômodos noturnos, recomendar banho quente e ambiente sereno, não realizar esforços intelectuais notáveis antes de dormir ${ }^{7}$. Quando o medicamento for prescrito, usar dose mínima e eficaz, efetuar tratamentos curtos (período menor de 2 meses) e intermitentes, suspender a medicação assim que os sintomas forem controlados ${ }^{6}$.

A orientação médica relacionada ao uso dos BZD é um fator muito importante para minimizar a incidência dos efeitos colaterais, além de propiciar maior autonomia para a percepção do risco e da dependência ${ }^{5}$. Deve ser fortemente enfatizado o poder de dependência da droga, diminuição da atividade motora, prejuízo da memória, tolerância e possível interação com outras drogas depressoras ${ }^{12}$.

Outras vezes, muitos pacientes vão ao médico, somente para obter nova prescrição, fonte primária de suprimento pelas pessoas que usam abusivamente esse tipo de droga, momento que o médico generalista e a equipe de saúde possuem papel fundamental na identificação de tais casos ${ }^{1}$.

Quando for o caso de uma prescrição nova, esta deve ser feita utilizando racionalidade, pois o uso dessa droga vem sendo propagado por médicos que muitas vezes não têm o conhecimento suficiente de psicofarmacologia e não seguem as recomendações de indicar drogas de meia vida prolongada para transtornos de ansiedade, e curta para a insônia, o que resulta em efeito residual ${ }^{6,7}$. Reflexo disso, é o fato de a maioria das prescrições de BZD (71,2\%) serem feitas em unidades primárias de saúde por não especialistas $^{12}$. 
É importante avaliar qual foi o problema que indicou a prescrição e se já houve a tentativa para o abandono da droga, momento em que se devem avaliar, também, os sinais da síndrome de abstinência'. Os transtornos que com maior frequência se associam à dependência são: depressão, ansiedade e transtornos de personalidade ${ }^{3}$.

A dependência deve ser amplamente prevenida com uso de dosagens mínimas e por períodos de tratamento o mais curto possível e seleção do paciente evitando prescrever esse tipo de medicamento a paciente propenso a drogadição como adultos com antecedente de alcoolismo ${ }^{1,6}$. Quando instalada, deve determinarse o grau de dependência de cada paciente identificando casos de maior ou menor gravidade $^{6}$. Segundo Oliveira ${ }^{3}$ os principais fatores para severidade, são doses elevadas (três ou mais comprimidos diários), uso prolongado, e BZD de maior potência ou de meia vida maior como o diazepam. Não obstante, também é necessário discernir os sintomas da abstinência do reaparecimento dos sintomas da ansiedade, o que pode ser responsável pelo insucesso na tentativa da interrupção da droga ${ }^{7}$.

Quando indicado o abandono do medicamento, isso deve ser feito de forma gradual e cautelosa. Diminuir a dose diária em $10-15 \%$ a cada 2 semanas. Recomenda-se também a utilização de drogas que atenuem os sintomas de abstinência como a fluoxetina e os antidepressivos tricíclicos, além dos antipsicóticos e anti-histamínicos ${ }^{3}$.

Dentre os principais fatores para a redução do uso desses medicamentos estão a consciência da mudança de estilo de vida e o conhecimento de outras estratégias não farmacológicas que devem ser tentadas antes de iniciar o tratamento com benzodiazepínicos ${ }^{7}$. Uma vez iniciado, entretanto, deve-se fornecer claro aconselhamento da importância de se evitar seu uso prolongado ${ }^{5}$.

\section{CONCLUSÃO}

Conclui-se que se deve instituir o uso racional de benzodiazepínicos na população idosa, evitando terapêutica prolongada e drogas com principio ativo com meia vida longa. Verificase também a necessidade do conhecimento pelo médico em saber identificar tolerância e dependência e como manejar esses pacientes.

\section{CONFLITO DE INTERESSE}

Os autores declaram não haver qualquer potencial de conflito de interesse que possa interferir na imparcialidade deste trabalho científico.

\section{REFERÊNCIAS}

1. Huf G, Lopes CS, Rozenfeld S. O uso prolongado de benzodiazepínicos em mulheres de um centro de convivência para idosos. Cad Saúde Púb. 2000;16(2):351-62.

DOI:

http://dx.doi.org/10.1590/S0102-

\section{X2000000200006}

2. Auchewski L, Andreatini R, Galduróz JCF, Lacerda RB. Avaliação da orientação médica sobre os efeitos colaterais de benzodiazepínicos. Rev Bras Psiquiatr. 2004;26(1):24-31. DOI: http://dx.doi.org/10.1590/S151644462004000100008

3. Olivera VM. Dependencia a benzodiazepinas em un centro de atención primaria de salud: Magnituddel problema y orientaciones para el manejo integral. Rev Chil Neuro-psiquiatr. 2009;47(2):132-7.

DOI: http://dx.doi.org/10.4067/S0717-

\section{5}

4. Galleguillos U Tamara, Risco N Luis, Garay S José Luis, González A Marco, Vogel M. Melina. Tendencia del uso de benzodiazepinas en una muestra de consultantes enatención primaria. Rev Méd Chile. 2003;131(5):535-40.

5. Souza ARL, Opaleye ES, Noto AR. Contextos e padrões do uso indevido de benzodiazepínicos entre mulheres. Ciên Saúde Col. 2013;18(4):113140.

6. Minaya O, Ugalde O, Fresán A. Uso inapropiado de fármacos de prescripción: dependencia a benzodiazepinas en adultos mayores. Salud Ment. 2009;32(5):405-11.

7. Fernández García A, González Viña A, Penã Machado MA. Bases científicas para el uso de lasbenzodiazepinas. Rev Cubana Med Gen Integr.2003;19(1).

8. Mendes KDS, Silveira RCCP, Galvão CM. Revisão integrativa: método de pesquisa para a incorporação de evidências na saúde e na enfermagem. Texto Contexto Enferm. 2008;17(4):758-64. DOI: http://dx.doi.org/10.1590/S010407072008000400018

9. Silva Herrera L, Vergara Fabián E, YeraAlós I, Freijoso E. Utilización de benzodiazepinas en la 
Atención Primaria de Salud. Rev Cubana Med Gen Integr. 2002;18(3):187-90.

10. Sánchez Ricardo LI, Hernández Gárciga FF. Consumo de benzodiazepinas en pacientes geriátricos Del Consultorio \#12, Policlínico "Campo Florido". Rev Cubana Farm. 2010;44(3):346-53.

11. Floriano PJ, Dalgalarrondo P. Saúde mental, qualidade de vida e religião em idosos de um Programa de Saúde da Família. J Bras Psiquiatr. 2007;56(3):162-70.

DOI:

http://dx.doi.org/10.1590/S0047-

20852007000300002

12.Silva VP, Botti NCL, Oliveira VC, Guimarães EAA. Perfil epidemiólogico dos usuários de benzodiazepínicos na atenção primária à saúde. Rev Enferm Centro-Oeste Min. 2015;5(1):13931400.

Recebido para publicação em 01/07/2016

Revisado em 10/07/2016

Aceito em 14/07/2016 